Original article

\title{
Risk assessment of re-emerging Plasmodium falciparum on Ishigaki Island using a stochastic transmission model
}

\author{
Yuuki Nakagawa ${ }^{1, \dagger}$, Masao Ueki $^{2, \dagger}$, Kaoru Fueda $^{1}$, Hiroshi Ohmae ${ }^{3}$ and Hirofumi Ishikawa $a^{1, *}$ \\ Received 24 March, 2009 Accepted 13 June, 2009 Published Online 30 July, 2009
}

\begin{abstract}
On Ishigaki Island, Plasmodium falciparum and Plasmodium vivax epidemics occurred in 1945-1946 and were successfully suppressed. The epidemic re-emerged in 1949 because many settlers immigrated to the former endemic areas, but it terminated in 1961. The present study aimed at predicting an outbreak of a new epidemic based on the situation in which P. falciparum malaria patients stay on Ishigaki Island and also examined the reemergence of the P. falciparum epidemic in 1951-1960 to determine the reliability of the model.

A stochastic transmission model of $P$. falciparum was constructed to detect a small number of infected persons. The seasonal fluctuation of the Anopheles minimus population obtained by observational data and meteorological data through statistical processing was introduced into the model.

Simulations were carried out to predict the risk of a new epidemic with scenarios in which the attribute of index patient, visiting season, and reduced inoculation rates of An. minimus were assumed. When an infected person visited the island in summer, a small number of patients with primary infections derived from the index patient appeared for all 1,000-iterations. On the other hand, when an infected person visited the island in winter, few or no patients with primary infections appeared for any of the 1,000-iterations because of the low mosquito density. In realistic conditions, the simulation results showed that there was little possibility of the occurrence of $P$. falciparum infection.
\end{abstract}

Keywords: Plasmodium falciparum, Anopheles minimus, Ishigaki Island, re-emergence, stochastic simulation

\section{INTRODUCTION}

Ishigaki Island belongs to the Yaeyama Archipelago located in southwestern Japan. It is the center of local government and economic activity in the Yaeyama Archipelago and is also known as a marine resort. Formerly a malaria endemic area, Ishigaki Island suffered a Plasmodium falciparum and Plasmodium vivax epidemic in 1945-1946, with a more than $50 \%$ prevalence on the island $[1,2]$. Although suppressed successfully, the epidemic re-emerged in 1949 because many settlers immigrated to former endemic areas and finally terminated in 1961 [2]. Toma et al. [3, 4] showed the presence of Anopheles minimus, which is the unique vector of $P$. falciparum on Ishigaki Island [2]. It is feared that a $P$. falciparum epidemic may occur again as a consequence of global warming.

The purpose of the present study was to predict the outbreak of an epidemic as a result of a $P$. falciparum malaria patient visiting Ishigaki Island and also to examine the re-emergence of the P. falciparum epidemic in 1951-1960. A stochastic transmission model of $P$. falciparum was constructed to detect a small number of infected persons, where the incubation period, recovery period, and period for the loss of infectivity were assumed to be governed by appropriate probability-distributions. A stochastic model can deal with infectious status at an individual level, in contrast to deterministic models dealing with a homogeneous mass of infected people.

Since $P$. falciparum induces more severe symptoms than other human malarias, many studies have been conducted on transmission models of $P$. falciparum. Dietz et al. [5] suggested a transmission model of $P$. falciparum using vectorial capacity [6] to produce quantitative results. Collett \& Lye [7] developed the Dietz et al. model [5] to es-

\footnotetext{
'Department of Human Ecology, Graduate School of Environmental Science, Okayama University, Okayama, 700-8530, Japan

2 The Institute of Statistical Mathematics, Minato, Tokyo, 106-8569, Japan

Department of Parasitology, National Institute of Infectious Diseases, Shinjuku, Tokyo, 162-8640, Japan

*Corresponding author:

Department of Human Ecology, Graduate School of Environmental Science, Okayama University, Okayama, 700-8530, Japan

E-mail: ishikawa@ems.okayama-u.ac.jp

+ Present address: Glory Co., Himeji, 670-8567, Japan

${ }^{\dagger \dagger}$ Present address: Faculty of Medicine, Yamagata University, Yamagata, 990-9585, Japan
} 
timate the effect of malaria control measures in Malaysia. Ishikawa et al. [8] developed a model from Collett \& Lye [7] to estimate the effect of malaria control measures in Vanuatu. Nakazawa et al. [9] built a stochastic model of $P$. falciparum to estimate the effectiveness of preventative measures among villagers, and $\mathrm{Gu}$ et al. [10] carried out simulations of $P$. falciparum transmission using an individual-based model.

Assessment of the seasonal fluctuation of the An. minimus population on Ishigaki Island was obtained from observational data on the density of immature An. minimus [4] combined with meteorological data [11] through statistical processing. In addition, the inoculation rate of humans, which reflects vectorial capacity [6], was estimated by the collection of data on An. minimus by human bites [12] and the above seasonal fluctuations.

The situation of the re-emergence of $P$. falciparum in the former Ohama Village in the 1951-1960 endemic area was simulated with 1,000-iterations. The simulation results followed the time-course of re-emergence and subsequent suppression of P. falciparum prevalence in the Yaeyama Archipelago.

To predict the risk of an epidemic when a $P$. falciparum malaria patient visits Ishigaki Island, several scenarios were prepared. When an infected person visited the island in summer, a small number of patients with primary infections that were derived from the index patient appeared for all 1,000-iteration simulations. On the other hand, when an infected person visited the island in winter, few or no patients with primary infections appeared for any of the 1,000-iteration simulations because of the low mosquito density. In realistic conditions, the simulation results showed that there was little possibility of an occurrence of P. falciparum infection.

\section{MATERIALS AND METHODS}

\section{Study area}

Belonging to the Yaeyama Archipelago, Ishigaki Island has an area of $222.63 \mathrm{~km}^{2}$ [13] and is located at $24^{\circ} 20^{\prime}-$ $36^{\prime} \mathrm{N}, 124^{\circ} 04^{\prime}-20^{\prime} \mathrm{E}, 411 \mathrm{~km}$ and $277 \mathrm{~km}$ from Naha (Okinawa) and Taipei (Taiwan), respectively [14]. The yearly average temperature and annual rainfall observed in 2007 were $24.8^{\circ} \mathrm{C}$ and $2270.0 \mathrm{~mm}$, respectively [11]. The daily temperature changes only slightly throughout the year. Previously, Toma et al. [3, 4] showed the presence of An. mini$m u s$, which is the unique vector of $P$. falciparum on Ishigaki Island [2].

The selected study area for the re-emergence of $P$. falciparum in the 1950s was the former Ohama Village in eastern Ishigaki Island, where malaria was endemic, and
Arakawa Town, a popular area for tourists in southern Ishigaki Island, was selected as a future invasion area of $P$. falciparum.

\section{Tourists on Ishigaki Island}

In recent years, many tourists visit Ishigaki Island in connection with its fame as a movie set, as a result of advertising, or on school trips. Tourists may play a key role as carriers in the invasion of $P$. falciparum to Ishigaki Island. The number of tourists visiting Ishigaki Island was reported to be about 767,000 in 2006, ranging between 50,000 (winter) and 80,000 (summer) a month [15]. The mean period of stay for tourists to the Yaeyama Archipelago in 2001 was estimated to be 2.8 days [16]. On the basis of the period of stay among tourists visiting Okinawa Prefecture in 2006 (mean 2.6 days) [17], the distribution of period of stay on Ishigaki Island was modified slightly from that for the whole of Okinawa Prefecture [18].

\section{Re-emergence of $P$. falciparum in 1951-1960}

The 1945-1946 P. falciparum and $P$. vivax epidemic in the Yaeyama Archipelago was successfully suppressed. However, it occurred again in 1949 because many settlers immigrated to the former endemic areas. The new epidemic terminated in 1961 [2, 19]. The occurrence of malaria patients in the Yaeyama Archipelago in the 1950s is shown in Fig. 1 [19]. The $P$. falciparum epidemic peaked earlier than the $P$. vivax epidemic.

Records show that 4,165 settlers immigrated to the Yaeyama Archipelago in 1949-1955 [20]. The change in the population of Ohama Village is based on the total number of settlers and demographic statistics in the 1950s [21], since no immigration data are available for Ohama Village in the $1950 \mathrm{~s}$.

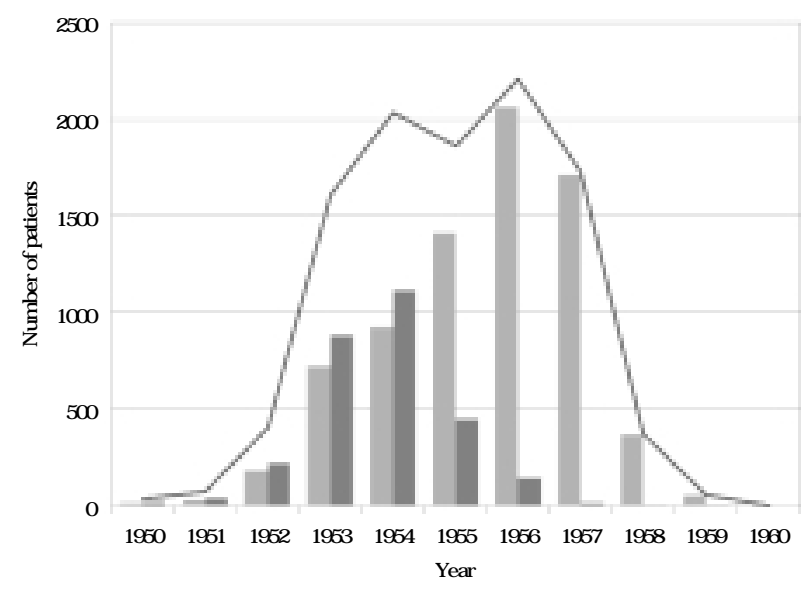

Fig. 1 The number of malaria patients in Yaeyama Archipelago (1950-1960).

Dark and light bars indicate the number of patients of $P$. falciparum and $P$. vivax, respectively, while the line indicates the total number of malaria patients. 


\section{Vector density}

Toma et al. [3, 4] observed the seasonal fluctuation of An. minimus in Nishihama Stream in the former Ohama Village, the study area for re-emergence in the 1950s. In 1957, a new vector control method, i.e., DDT residual spraying conducted drastically twice a year, was started. Consequently, no Anopheles mosquitoes were collected in 1961 mosquito surveys and malaria was eradicated in 1961 $[2,22]$. The seasonal fluctuation of the An. minimus population in the 1950s was estimated by the generalized linear model in mathematical statistics through observation data on the density of immature An. minimus [4], collected data

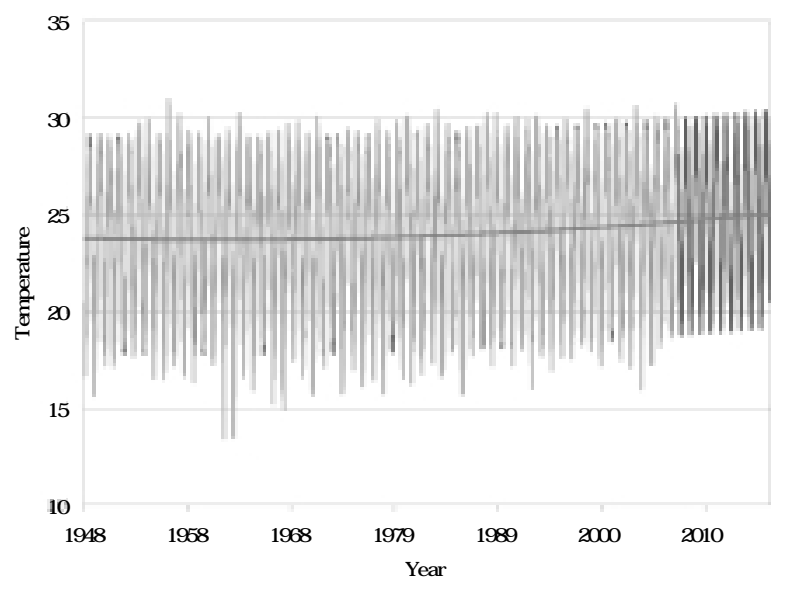

Fig. 2 The observed and estimated temperature on Ishigaki Island.

The light line indicates the observed temperature (1948-2007) and the dark line indicates the estimated temperature (2007-2016). The grey bold line indicates the estimated quadratic curve of the trend in temperature.

(a)

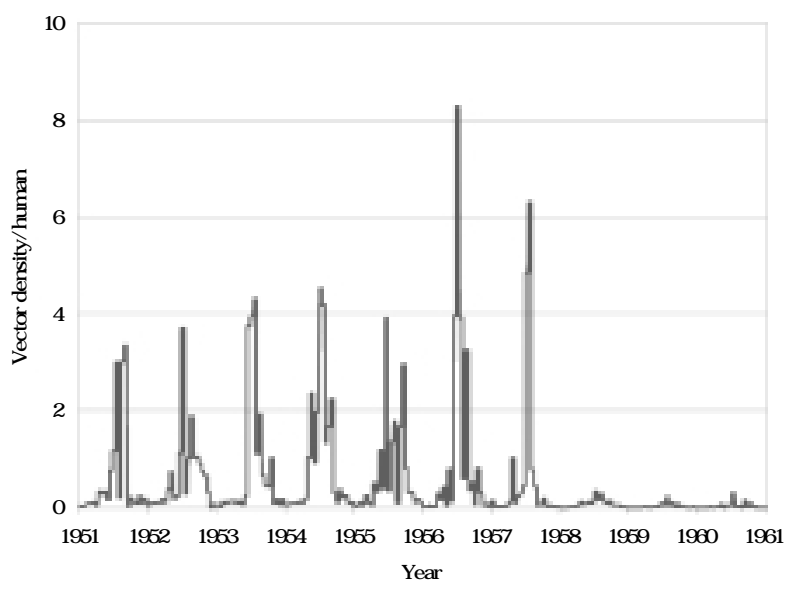

on human bites [12], and meteorological data [11], which would be reduced to 5-20\% due to DDT residual spray after August 1957. The vector density correlated with water temperature, which in turn correlated with air temperature and rainfall [4].

To predict future temperature and rainfall changes which will reflect the global warming trend of recent years, the records on temperature and rainfall for the past 60 years (1948-2007) at Ishigaki Island were divided into seasonality, trend and noise components using standard methods in time series analysis. By the hypothesis testing, which detected an increase trend in temperature but not in rainfall, future temperature changes had trend and seasonality (Fig. 2), while future rainfall changes had only seasonality. The details were given by Ueki et al. [18]. The future density of An. minimus per person in 2008-2017 was estimated by the same way as the 1951-1960 period, except for the effect of DDT residual spraying.

The estimated densities of An. minimus per person in 1951-1960 and in 2008-2017 on Ishigaki Island are shown in Fig. 3 (a) and (b), respectively.

\section{Mathematical model Model Scheme}

In the present study, a stochastic model was constructed for $P$. falciparum transmission. In this model, the human population was divided into five epidemiological classes: negative, preventive, incubation, positive, and protective. The preventive class was comprised of people receiving preventative treatment against malaria, while the protective class was comprised people under treatment for malaria. The injection of $P$. falciparum by infected vectors transferred an individual from the negative class to the incu-

(b)

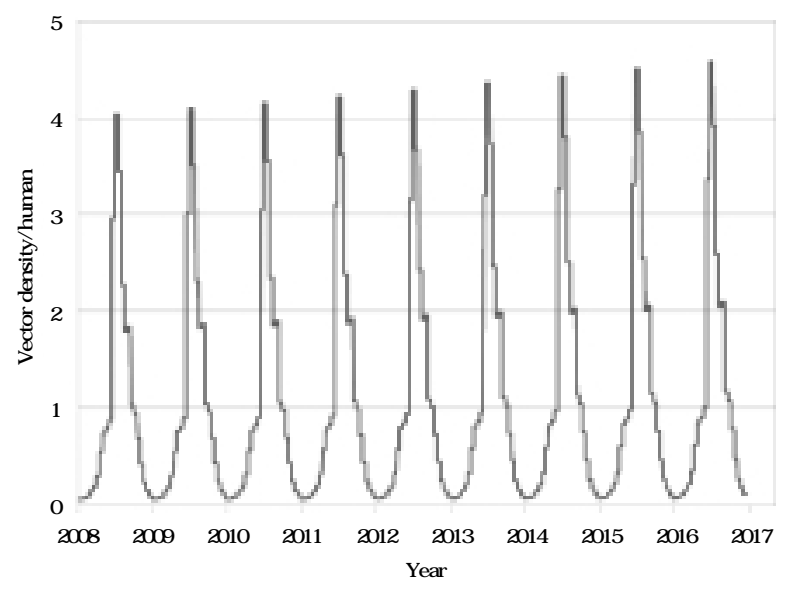

Fig. 3 The estimated densities of An. minimus per person on Ishigaki Island.

(a) 1951-1960, (b) 2008-2017 
bation class. After the incubation period, the individual showed parasitemia and suffered clinical symptoms such as fever. Repeated proliferation of parasites in erythrocytes brings on fever, and some parasites in erythrocytes differentiate into gametocytes. The positive class was subdivided into two subclasses: a positive and infectious subclass with gametocytes (PG) and a positive but not infectious subclass without gametocytes (PN). The appearance of gametocytes transferred an individual from the PN-subclass to the PGsubclass, while the disappearance of gametocytes transferred an individual from the PG-subclass to the PNsubclass. The protective class was also subdivided into two subclasses: a protective and infectious subclass (PrG) and a protective but not infectious subclass $(\operatorname{PrN})$. The disappearance of asexual parasites by the effect of chemotherapeutic treatment transferred an individual from the PN and PGsubclasses to the PrN and PrG-subclasses, respectively, while the disappearance of gametocytes by the effect of chemotherapeutic treatment transferred an individual from the PrG-subclass to the PrN-subclass. Spontaneous recovery without treatment transferred an individual from the PN-subclass to the negative class. The interruption of medication transferred an individual from the PrG-subclass to the PN-subclass. An individual discharged from the hospital after complete recovery was transferred from the PrNsubclass to the negative class. After 1954, prevention by prophylactic treatment against malaria transferred an individual from the negative or incubation class to the preventive class, and the interruption of prevention transferred an individual from the preventive class to the negative class.

In the projected situation of a future invasion of $P$. falciparum, all patients will go to hospital and be diagnosed, so no spontaneous recovery without treatment was taken into account. An individual of the PN-subclass transferred not to the negative class but to the PG or PrN-subclasses. In addition, the interruption of medication and prevention were not considered. Since a patient was assumed to be in the hospital after diagnosis, he or she would no longer be subject to mosquito bites.

Two model schemes for $P$. falciparum transmission on Ishigaki Island, one in the 1950s situation and the other in the future invasion situation, are shown in Fig. 4 (a) and (b), respectively.

\section{Estimation of parameters in the model}

In order to carry out stochastic simulations, it is necessary to include epidemiological parameters etc. to determine suitable probability-distributions with means and variances on the basis of field data.

\section{Prevention}

The transfer of an individual from the negative or incubation class to the preventive class under prophylactic treatment was assumed to be governed by a Bernoulli distribution with a low success rate of 0.005 because the prophylactic treatment resulted in failure [23], and the duration of prophylactic treatment was assumed to be governed by a Gamma distribution with a mean of 10.5 days and a variance of $3.5^{2}$.

\section{Incubation period}

Individuals injected with an infected vector showed clinical symptoms after 1 week at the earliest and most of them within a month [24]. The incubation period, that is, the appearance of parasites after infection, was assumed to be governed by a Gamma distribution with a mean of 10.52 days and a variance of $0.17^{2}$ [25].

\section{Period for loss of infectivity}

The period for the loss of infectivity was assumed to be governed by a Gamma distribution with a mean of 19.5 days and a variance of $4.0^{2}$ [26].

\section{Recurrence}

In the $1950 \mathrm{~s}$, the recurrence rate of $P$. falciparum in the PrN- PrG-subclasses was assumed to be governed by a Bernoulli distribution with a success rate of 0.08 [27].

Out of the hospital

The transfer of an individual from the PrN-subclass to the negative class in the 1950s was assumed to be governed by a Bernoulli distribution with a success rate of 0.05 due to (a)

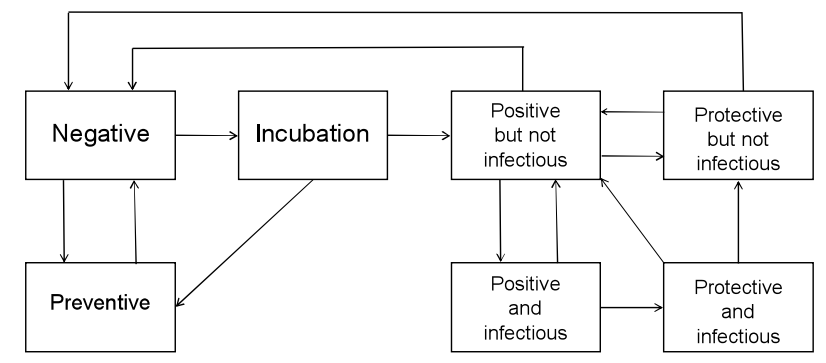

(b)

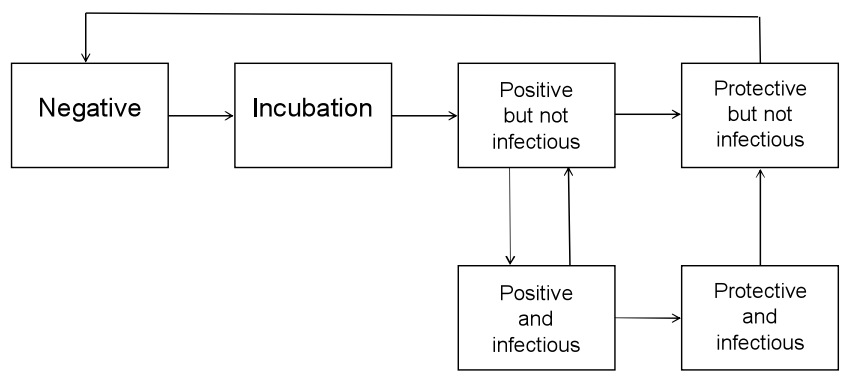

Fig. 4 Model schemes for P. falciparum transmission on Ishigaki Island.

(a) for the situation in the $1950 \mathrm{~s}$, (b) for the situation of a future invasion 
a lack of field data. On the other hand, for the situation of a future invasion, an individual in the PrN-subclass was assumed to transfer to the negative class after 17 dayshospitalization.

\section{Recovery period without treatment}

The recovery period without treatment in the 1950s was assumed to be governed by a Gamma distribution with a mean of 250 days and a variance of $50^{2}$ [28].

\section{Recovery period under treatment}

On Ishigaki Island in the 1950s, the drugs atebrin and plasmochin were used for malaria treatment. The recovery period with these drugs was assumed to be governed by an exponential distribution with a mean of 1.1 days and 3.5 days for asexual parasites and gametocytes, respectively.

In the situation of a future invasion of $P$. falciparum, it was assumed that a three-day course of Artemetherlumefantrine $(\mathrm{AL})$, which is a type of Artemisinin-based combination therapy (ACT), will be adopted for malaria treatment [29]. The period for the loss of infectivity was assumed to be governed by a Gamma distribution with a mean of 19.5 days and a variance of $4.0^{2}$ [26].

Efficacy of drugs

In the situation of a future invasion of $P$. falciparum, it was assumed that $96 \%$ of treated patients show an adequate clinical response [30]. The transition rate from both the PN and PrG-subclasses to the PrN-subclass was governed by a Bernoulli distribution with a success rate of 0.96 .

\section{Inoculation}

The inoculation of $P$. falciparum by mosquitoes was assumed to be governed by a Bernoulli distribution with a success rate of $g(1-\exp (-C Y))$ [5], where $C, g$, and $Y$ stand for the vectorial capacity as described by Garrett-Jones [6], the susceptibility as a conditional probability of infection as described by Dietz et al. [5], and the ratio of the PG and PrG-subclasses. People in the PN and PG-subclasses were assumed to have a risk of super-infection, and superinfection was governed by a Bernoulli distribution with the same success rate as above.

Other parameters

The rate of acquisition of infectivity $(\alpha)$ and susceptibility $(g$ ) cannot be determined by field or experimental data. A value satisfying the prevalence rate of $50 \%$ was chosen [1], and the number of infectious patients was equal to the number of noninfectious patients $[31,32]$. A value of $\alpha=0.025$ was chosen, and $g=0.006$ as the minimum of $\chi^{2}$ was recorded as 0.41 . Here, $\chi^{2}$ stands for the formula: $\chi^{2}=$ $\left(\mathrm{Y}_{1} / \mathrm{Y}_{2}-1\right)^{2}+\left(\mathrm{X}_{1} / \mathrm{X}_{2}-1\right)^{2}$, with $\mathrm{X}_{1}$ : negative class and incubation class, $\mathrm{X}_{2}$ : Positive class, $\mathrm{Y}_{1}$ : $\mathrm{PN}$-subclass, and $\mathrm{Y}_{2}$ : PGsubclass.

The estimated values and the types of distribution of these parameters are presented in Table 1 .

\section{Scenarios}

Scenario in the situation of a future invasion

In the situation of a future invasion, two kinds of index case - resident and tourist - and two visiting season - high (July) and low (January) mosquito-density seasons - were presumed. The period for diagnosis was governed by a $\chi^{2}$ distribution with degrees of freedom of 20 and 4.7, which stand for the mean period for diagnosis, within a month of index case's visiting, and thereafter, respectively [33]. The scenarios are summarized in Table 2.

Inoculation rates in the situation of a future invasion

The inoculation rate could decrease with the spread of air conditioners and urbanization. Therefore, various inoculation rates (relative inoculation rate (RI)) reduced to $25 \%$, $50 \%$, and $100 \%$ compared with the level of An. minimus density in the 1950 s were modelled.

(b)

\begin{tabular}{ccc}
\hline Rate & Type of distribution & Success rate \\
\hline Prevention & Bernoulli & 0.005 \\
Recurrence $(1950 \mathrm{~s})$ & Bernoulli & 0.08 \\
Out of the hospital & Bernoulli & 0.05 \\
Adequate clinical response & Bernoulli & 0.96 \\
Acquisiton of infectivity & Bernoulli & 0.025 \\
\hline
\end{tabular}

(c)

\begin{tabular}{ccc}
\hline Description (rate) & Symbol & Value \\
\hline Susceptibility & $g$ & 0.006 \\
\hline
\end{tabular}

(a)

\begin{tabular}{ccccc}
\hline Period & $\begin{array}{c}\text { Type of } \\
\text { distribution }\end{array}$ & $\begin{array}{c}\text { Mean } \\
\text { (days) }\end{array}$ & Variance & $\begin{array}{c}\text { Range } \\
\text { (days) }\end{array}$ \\
\hline Incubation & Gamma & 10.52 & $0.17^{2}$ & $9-14$ \\
Recovery & Gamma & 250 & $50^{2}$ & $200-300$ \\
Loss of infectivity & Gamma & 19.5 & $4^{2}$ & $16-24$ \\
Prophylactic & Gamma & 10.5 & $3.5^{2}$ & $7-14$ \\
Recovery from & & & & \\
parasitemia & Exponential & 1.1 & - & - \\
by atebrin and & & & & \\
plasmochin & & & & - \\
Recovery from & & & & \\
parasitemia with & & & & \\
gametocytes & Exponential & 3.5 & & \\
by atebrin and & & & & \\
plasmochin & & & & \\
\hline
\end{tabular}


Table 2 Scenarios for the situation of a future invasion.

\begin{tabular}{|c|c|c|c|c|c|}
\hline \multirow[b]{2}{*}{ Scenario } & \multirow{2}{*}{$\begin{array}{l}\text { Relative } \\
\text { inoculation } \\
\text { rate }(\mathrm{RI})\end{array}$} & \multicolumn{3}{|c|}{ Index case } & \multirow{2}{*}{$\begin{array}{c}\text { Period for diagnosis } \\
\text { Probability-distributions } \\
\text { and mean (days) }\end{array}$} \\
\hline & & Attribution & $\begin{array}{l}\text { Visiting } \\
\text { season }\end{array}$ & Number & \\
\hline & 1 & & & & \multirow{5}{*}{$\begin{array}{c}\chi^{2} \text {-distribution } \\
20 \text { (First month) } \\
4.7 \text { (Second month) }\end{array}$} \\
\hline 1 & $\begin{array}{c}0.5 \\
0.25\end{array}$ & Resident & July & 1 & \\
\hline 2 & $\begin{array}{c}1 \\
0.5 \\
0.25\end{array}$ & Tourist & July & 1 & \\
\hline 3 & $\begin{array}{c}1 \\
0.5 \\
0.25\end{array}$ & Resident & January & 1 & \\
\hline 4 & $\begin{array}{c}1 \\
0.5 \\
0.25\end{array}$ & Tourist & January & 1 & \\
\hline
\end{tabular}

\section{RESULTS}

\section{Stochastic simulation}

The stochastic model of $P$. falciparum transmission was programmed by Intel Visual Fortran ${ }^{\mathrm{TM}}$ to work on any computer using the Microsoft Windows ${ }^{\mathrm{TM}}$ platform, where the random number generators in IMSL $^{\mathrm{TM}}$ libraries were used. With regard to the method of simulation, the time step of the stochastic process was adopted as a day, and the results in this section were obtained through 1,000-iteration simulations.

\section{Simulations}

Simulation of the re-emergence of $P$. falciparum in 19511960

The situation of the re-emergence of $P$. falciparum in

(a)

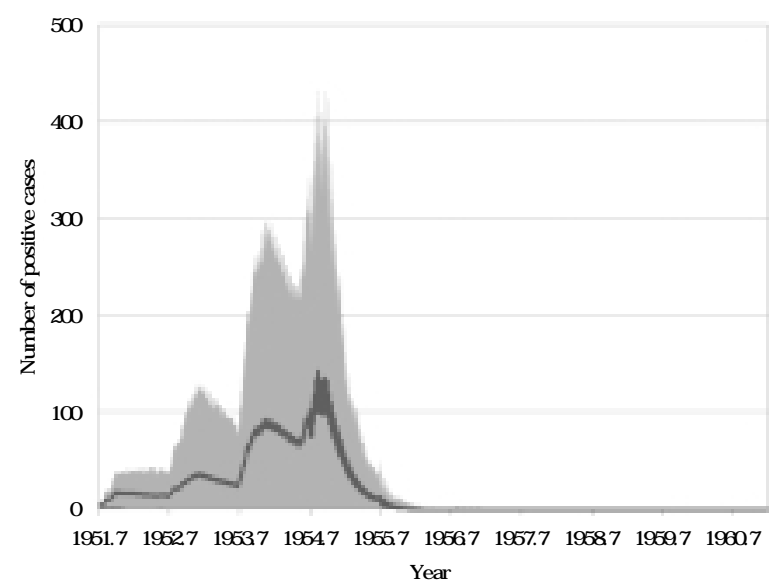

Table 3 Assumption of the frequency and coverage of prophylactic and chemotherapeutic treatments.

\begin{tabular}{cccc}
\hline Year & $\begin{array}{c}\text { Frequency } \\
\text { of treatment }\end{array}$ & $\begin{array}{c}\text { Coverage of } \\
\text { chemotherapeutic } \\
\text { treatment (\%) }\end{array}$ & $\begin{array}{c}\text { Coverage of } \\
\text { prophylactic } \\
\text { treatment (\%) }\end{array}$ \\
\hline $1951-1953$ & 2 & 10 & - \\
1954 & 3 & 30 & 0.5 \\
$1955-1960$ & 3 & 70 & 0.5 \\
\hline
\end{tabular}

the former Ohama Village of the previous endemic area in 1951-1960 was simulated stochastically using the model. In the simulation, the initial population in 1951 was assessed as 5,519 by demographic data from 1947 [34], and the population increased due to immigration (2.1.2). Five initial patients were introduced to the targeted village, but the exact number remained unexplained. For the situation in the 1950s on Ishigaki Island, it was assumed that prophy-

(b)

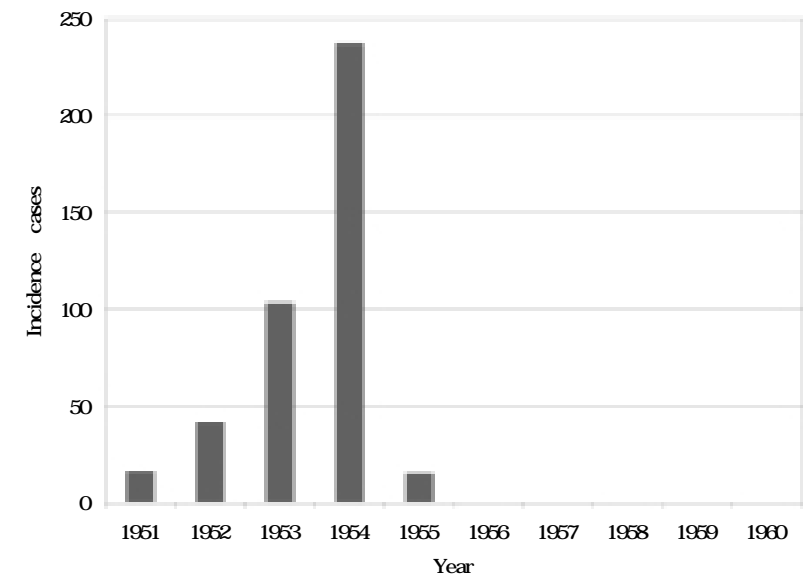

Fig. 5 Simulations of re-emergence of $P$. falciparum in 1951-1960.

(a) The number of patients in 1951-1960 in 1,000-iteration simulations.

The black line indicates the profile of the mean numbers, and the dark gray and light gray zones indicate standard derivation range and the maximum-minimum range. (b) The average yearly incidence in 1951-1960 in 1000-iteration simulations. 
(a)

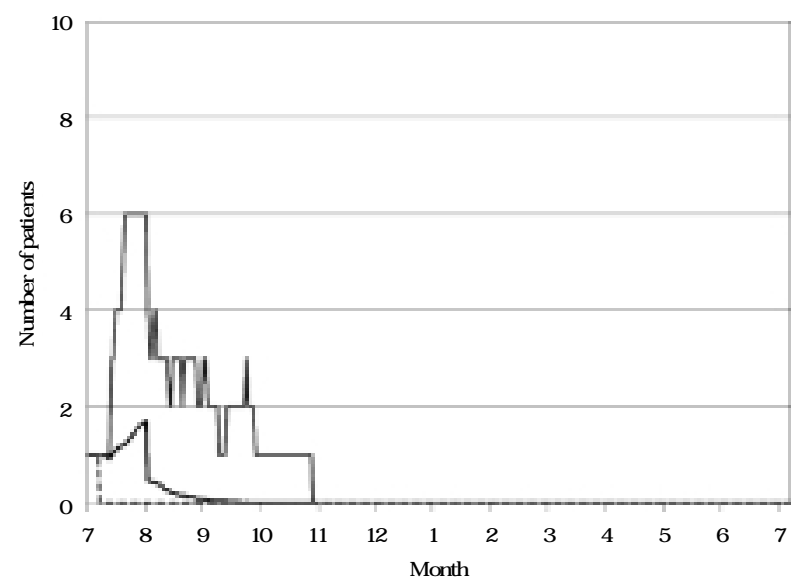

(c)

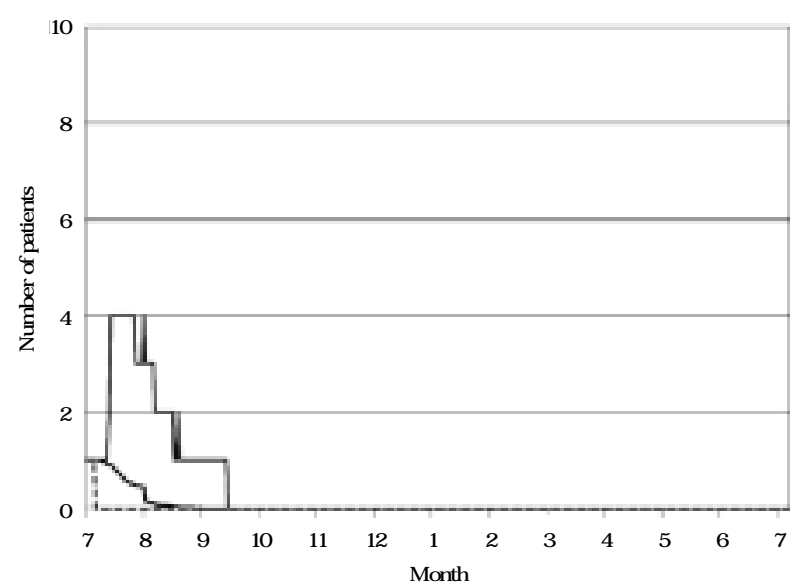

(e)

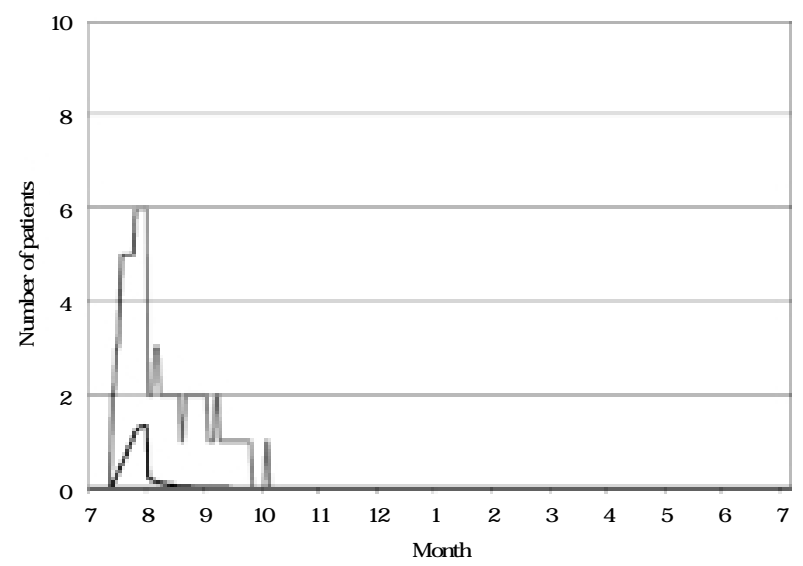

(b)

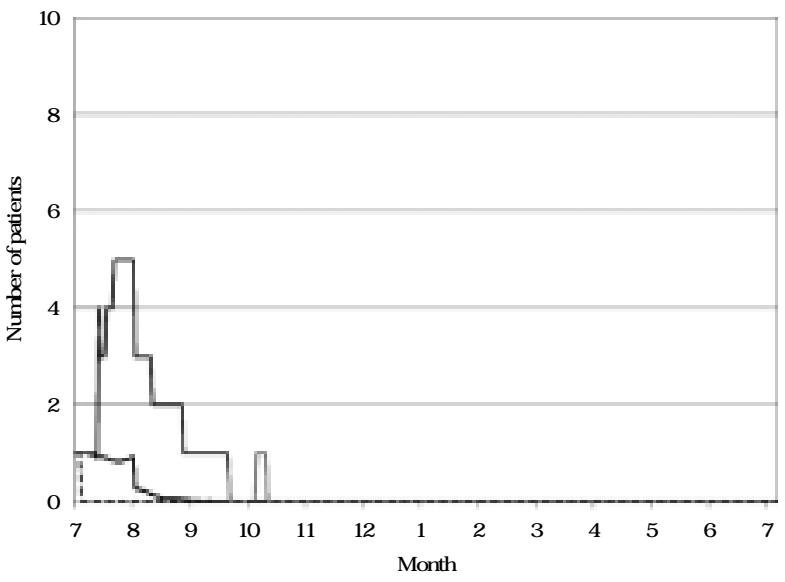

(d)

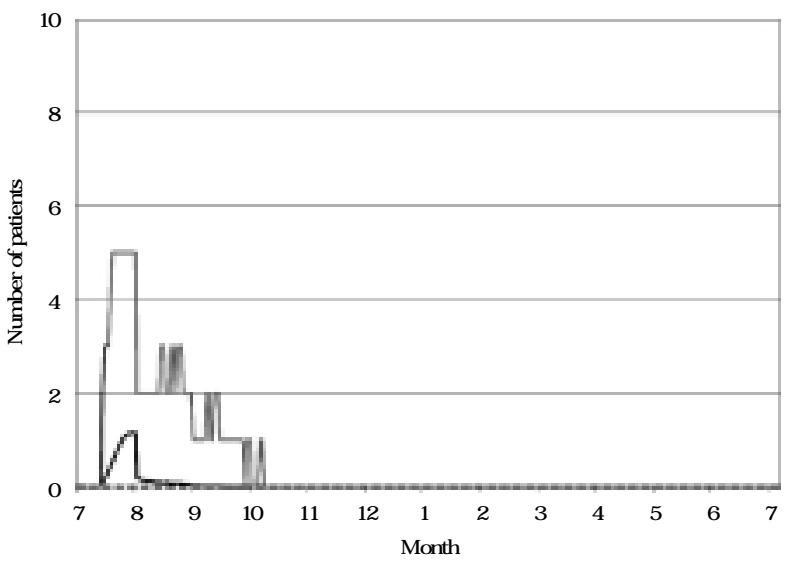

(f)

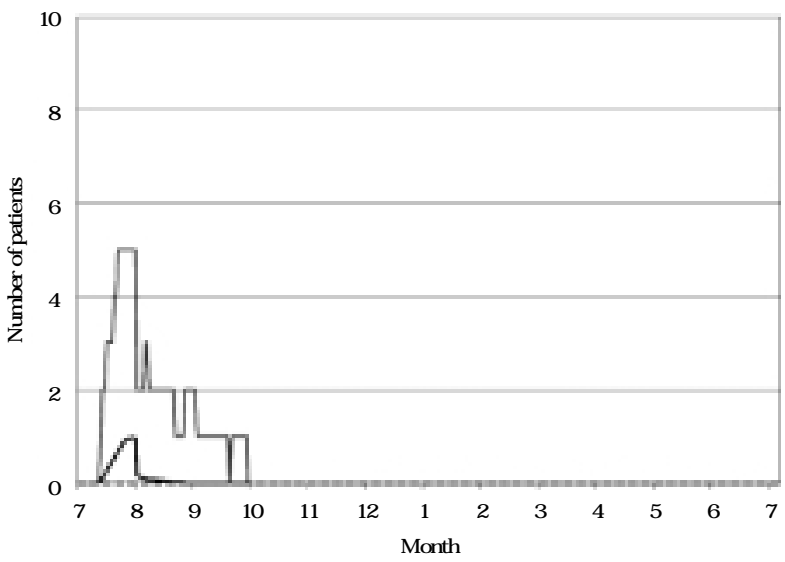

Fig. 6 The profile of the number of P. falciparum patients in scenarios (Table 2) in 1,000-iteration simulations.

The gray and black straight lines indicate the profile of the maximum numbers, the mean numbers, and the dark gray broken line indicates the profile of the minimum numbers in simulations. (a)-(c) and (d)-(f) show the situations based on scenarios 1 and 2 in Table 2, respectively, and (a,d), (b,e) and (c,f) show the relative inoculation rate (RI) of 1, 0.5, and 0.25, respectively. Figures for scenario 3 and 4 were omitted. 
lactic and chemotherapeutic treatments against malaria (2.3.2) were conducted twice a month from 1951 to 1953 and three times a month after 1954 [19, 23]. The coverage rate of prophylactic and chemotherapeutic treatments was assumed from descriptions in Yaeyama Regional Public Health and Welfare Division [19] and Eshita [23], as shown in Table 3, due to the lack of old full records. The introduction of DDT residual spray [22] was also considered (2.2). The profiles of the number of patients and number of yearly incidences are shown in Fig. 5 (a) and (b), respectively.

The simulation showed that the number of patients increased from 1951 to 1954 , reached at peak in 1954 with 150 patients as the mean and 480 patients as the maximum and decreased thereafter, no further patients being observed in 1957 in any of the trials.

Simulation of a future invasion of $\boldsymbol{P}$. falciparum patients

Simulations with 1,000 trials for 1 year were carried out for a future invasion of a $P$. falciparum patient with gametocytes with various scenarios (Table 2). In the model, the initial population was assessed as 8,772 by demographic data from 2006 [21] and the movement of tourists was in- troduced as 2.1.1. The profiles of the number of patients for 4 scenarios are shown in Fig. 6 (a)-(f). The number of patients among residents with primary infections that were derived from the index patient and the number of incidences among tourists are summarized in Tables 4 and 5, respectively. Tourists have little risk of infection and infected tourists seldom bring $P$. falciparum to any area other than Ishigaki Island, as shown by the fact that there were 2.98 infections on average and 11 infections maximum among tourists in the most serious situation of $\mathrm{RI}=1.0$ in scenario 1 (Table 5). The probability that a $P$. falciparum epidemic will be terminated within 1, 3, and 6 months is shown in Table 6 . In scenario 3 with $\mathrm{RI}=1.0$ and scenario 4 with three levels of RI, however, simulations with 1,000-iterations showed that exceptional trials produced few patients with primary infections and that 1,000-iterations in the simulation for $\mathrm{RI}=0.5,0.25$ in scenario 3 produced no patients with primary infections. So these figures were omitted.

Table 4 The number of patients among residents with primary infections in 1000-iteration simulations.

\begin{tabular}{|c|c|c|c|c|c|c|c|}
\hline \multirow[b]{2}{*}{ Scenario } & \multirow{2}{*}{$\begin{array}{c}\text { Relative } \\
\text { inoculation } \\
\text { rate (RI) }\end{array}$} & \multicolumn{6}{|c|}{ The number of patients with primary infections } \\
\hline & & Mean & Maximum & $\begin{array}{c}\text { Third } \\
\text { quartile }\end{array}$ & Median & $\begin{array}{c}\text { First } \\
\text { quartile }\end{array}$ & Minimum \\
\hline \multirow{3}{*}{1} & 1 & 24.96 & 70 & 34 & 22 & 11 & 0 \\
\hline & 0.5 & 12.82 & 55 & 22 & 11 & 0 & 0 \\
\hline & 0.25 & 5.69 & 22 & 11 & 0 & 0 & 0 \\
\hline \multirow{3}{*}{2} & 1 & 14.99 & 62 & 22 & 11 & 7 & 0 \\
\hline & 0.5 & 16.26 & 70 & 22 & 15 & 7.75 & 0 \\
\hline & 0.25 & 12.09 & 56 & 20 & 11 & 0 & 0 \\
\hline \multirow{3}{*}{3} & 1 & 0.19 & 11 & 0 & 0 & 0 & 0 \\
\hline & 0.5 & 0 & 0 & 0 & 0 & 0 & 0 \\
\hline & 0.25 & 0 & 0 & 0 & 0 & 0 & 0 \\
\hline \multirow{3}{*}{4} & 1 & 0.82 & 20 & 0 & 0 & 0 & 0 \\
\hline & 0.5 & 0.35 & 11 & 0 & 0 & 0 & 0 \\
\hline & 0.25 & 0.24 & 11 & 0 & 0 & 0 & 0 \\
\hline
\end{tabular}

Table 5 The incidence among tourists in 1000-iteration simulations.

\begin{tabular}{|c|c|c|c|c|c|c|c|}
\hline \multirow[b]{2}{*}{ Scenario } & \multirow{2}{*}{$\begin{array}{c}\text { Relative } \\
\text { inoculation } \\
\text { rate }(\mathrm{RI})\end{array}$} & \multicolumn{6}{|c|}{ Incidence } \\
\hline & & Mean & Maximum & $\begin{array}{c}\text { Third } \\
\text { quartile }\end{array}$ & Median & $\begin{array}{c}\text { First } \\
\text { quartile }\end{array}$ & Minimum \\
\hline \multirow{3}{*}{1} & 1 & 2.98 & 11 & 4 & 3 & 2 & 0 \\
\hline & 0.5 & 1.44 & 7 & 2 & 1 & 0 & 0 \\
\hline & 0.25 & 0.65 & 4 & 1 & 0 & 0 & 0 \\
\hline \multirow{3}{*}{2} & 1 & 1.73 & 9 & 3 & 1 & 1 & 0 \\
\hline & 0.5 & 1.79 & 8 & 3 & 2 & 1 & 0 \\
\hline & 0.25 & 1.3 & 7 & 2 & 1 & 0 & 0 \\
\hline \multirow{3}{*}{3} & 1 & 0.02 & 1 & 0 & 0 & 0 & 0 \\
\hline & 0.5 & 0 & 0 & 0 & 0 & 0 & 0 \\
\hline & 0.25 & 0 & 0 & 0 & 0 & 0 & 0 \\
\hline \multirow{3}{*}{4} & 1 & 0.09 & 2 & 0 & 0 & 0 & 0 \\
\hline & 0.5 & 0.04 & 1 & 0 & 0 & 0 & 0 \\
\hline & 0.25 & 0.02 & 1 & 0 & 0 & 0 & 0 \\
\hline
\end{tabular}


Table 6 Proportions of extinction.

\begin{tabular}{ccccc}
\hline \multirow{2}{*}{ Scenario } & $\begin{array}{c}\text { Relative } \\
\text { inoculation } \\
\text { rate (RI) }\end{array}$ & $\begin{array}{c}\text { Within 1 } \\
\text { month }\end{array}$ & $\begin{array}{c}\text { Within 3 } \\
\text { months }\end{array}$ & $\begin{array}{c}\text { Within 6 } \\
\text { months }\end{array}$ \\
\cline { 3 - 5 } & 1 & 25.6 & 99.2 & 100 \\
1 & 0.5 & 49.8 & 99.9 & 100 \\
& 0.25 & 71.3 & 100 & - \\
\hline \multirow{3}{*}{2} & 1 & 57.6 & 99.9 & 100 \\
& 0.5 & 61.3 & 99.9 & 100 \\
& 0.25 & 70.9 & 100 & - \\
\hline \multirow{3}{*}{3} & 1 & 94.8 & 100 & - \\
& 0.5 & 96.0 & 100 & - \\
& 0.25 & 94.9 & 100 & - \\
\hline \multirow{2}{*}{4} & 1 & 97.5 & 100 & - \\
& 0.5 & 99.2 & 100 & - \\
\hline
\end{tabular}

\section{DISCUSSION}

The epidemics of $P$. falciparum and $P$. vivax that occurred on Ishigaki Island in 1945-1946 were successfully suppressed. However, the epidemic occurred again in 1949 because many settlers immigrated to the former endemic areas. Malaria control programs beginning in 1947 were mostly ineffective in suppressing the epidemic. The eradication programs were revised in 1947 and again in 1957 [2, 23], and the epidemics of $P$. falciparum and $P$. vivax were terminated in 1961 [19]. A mathematical model of $P$. falciparum transmission was constructed and simulations were carried out following scenarios (3.2.1) to review the reemergence of P. falciparum in the 1950s on Ishigaki Island. Moreover, the risk of re-emergence of $P$. falciparum when a $P$. falciparum malaria patient visits Ishigaki Island was predicted through model simulations following further scenarios (2.4), because the continuing presence of An. minimus on Ishigaki Island has been reported by Toma et al. [3, 4].

The phenomenon of inoculations of $P$. falciparum into individuals by mosquitoes was assumed to be governed by a Bernoulli distribution with a success rate following the formula: $g(1-\exp (-C Y))(2.3 .2)$, the basis of which was introduced by Dietz et al. [5]. All of the epidemiological and entomological parameters in the model, except for the rate of acquisition of infectivity $(\alpha)$ and susceptibility $(g)$, were estimated on the basis of malariological surveys. Because the process of gametocyte production has not as yet been ascertained [35], it is difficult to determine the rate of acquisition of infectivity as an accurate value. In addition, susceptibility cannot be determined by experimental methods or field surveys. The rate of acquisition of infectivity and susceptibility were chosen to satisfy the conditions that the number of infectious patients would be equal to half the number of infectious patients $[31,32]$ and that the preva- lence rate would be $50 \%$ [1].

The simulation examining the re-emergence of $P$. falciparum in 1951-1960 (Fig. 5, (a)) showed that the number of patients increased gradually and reached a peak in 1954, and that the epidemic terminated in 1960 because of the effects of enhanced malaria control from 1954 (Table 3) and DDT residual spray from 1957 [2], factors that were brought into the assumptions of the scenario. The profile of the incidence obtained by the simulation (Fig. 5) was compared to that in the Yaeyama Archipelago in the 1950s (Fig. 1). Although there are still few details known about the situation in the 1950s in the former Ohama Village, the simulation results followed the time-course of re-emergence and the subsequent suppression of $P$. falciparum, which led to the transmission model being regarded as sufficiently reliable.

The inoculation rate was reduced by the improvement of public health, urbanization, and the spread of air conditioners [36]. Therefore, scenarios were arranged with reduced inoculation rates of $25 \%$ and $50 \%$ ( $\mathrm{RI}=0.25$ and 0.5 ) compared with the level of An. minimus density in the 1950 s $(\mathrm{RI}=1.0)$. The supposition that $\mathrm{RI}=0.25$ was better reflected the present conditions on Ishigaki Island.

In all scenarios, the diagnosis period was set at 20 days as the mean within the first month after the index case visits Ishigaki Island and 4.7 days thereafter. Christen [33] and Chalumeau et al. [37] reported that a mean of 4.7 days was required to diagnose $P$. falciparum in Europe, a nonendemic region. On the other hand, Chalumeau et al. [37] reported that it took a maximum of 19 days to diagnose $P$. falciparum. Since an epidemic of $P$. falciparum has not occurred for about 40 years on Ishigaki Island, it could take several days to reach a diagnosis of $P$. falciparum correctly at the beginning of the outbreak from the viewpoint of the risk assessment for $P$. falciparum.

From the viewpoint of risk assessment of an epidemic, the present study compared the two seasons when an infected person visited the island, that is, scenarios 1 and 2 (July, summer) and scenarios 3 and 4 (January, winter). In scenarios 1 and 2, a mean of 5-24 patients with primary infections derived from the index patient appeared for all 1,000-iterations, while in scenarios 3 and 4, few or no patients with primary infections appeared for any of the 1,000-iterations because of the low mosquito density (Table 4). In scenarios 1 and 2, some of the patients appeared just after the introduction of the index patients in summer because of the high mosquito density, and the epidemic terminated in autumn for all 1,000-iterations because of the low mosquito density (Table 6; Fig. 3 (b)).

The influence of a decrease in the inoculation rate on the occurrence of patients among residents and tourists and 
the proportion of extinction were investigated. The number of patients with primary infections derived from the index patient, together with the incidence among tourists, and probabilities of extinction decreased in correlation to the RI in all scenarios except several inverted value sets, which were insignificant (significance level, 0.05): P-value $=41 \%$ (Table 4, scenario 2, RI=1, 0.5, mean), 49\% (Table 5, scenario $1, \mathrm{RI}=1,0.5$, mean), $46 \%$ (Table 6 , scenario $3, \mathrm{RI}=0.5$, 0.25 , within 1 month).

In Taiwan, one and two patients were recognized as having autochthonous $P$. falciparum infection in 2001 and 2003, respectively, and there were 258 patients of imported P. falciparum infection during 1999-2008 [38]. Meanwhile, 61-83 persons infected with $P$. falciparum malaria abroad enter Japan every year (2002-2006) [39]. Although there is a low possibility of the occurrence of $P$. falciparum infection in the situation where $\mathrm{RI}=0.25$ (3.2.2), global warming may accelerate the expansion of the habitat of An. minimus and consequently the occurrence of $P$. falciparum infection. Therefore, it is necessary to establish a continuous monitoring system for the detection of $P$. falciparum malaria infection and surveillance for An. minimus.

\section{ACKNOWLEDGEMENTS}

This work was supported in part by a Grant-in-Aid from the Ministry of Health, Labour and Welfare of Japan (H20-Sinkou-ippan-015), a Grant-in-Aid from the Transdisciplinary Research Integration Center, the Research Organization of Information and Systems (A996130 to M.U.), a Grant-in-Aid from the Japan Society for the Promotion of Science (21540129 to H.I.) and a Grant-in-Aid from the Ministry of the Environment (S-4 to H.O.)

\section{REFERENCES}

1 . Department of Public Health, Yaeyama Civil Government. Department of Public Health and Malaria eradication. In: Memorial magazine for the new Yaeyama Exposition, Yaeyama Civil Government memorial magazine editorial office, 1950. In: The history in Ishigaki City, Ishigaki City Government, Ishigaki City, 1989: 736-752 (in Japanese).

2 . Miyagi I., Toma T., Malenganisho W., Uza M. Historical review of mosquito control as a component of malaria eradication program in the Ryukyu Archipelago. Southeast Asian Journal of Tropical Medicine and Public Health, 1996; 27: 498-511.

3 . Toma T., Miyagi I., Malenganisho W. L. M., Tamashiro M., Takagi M., Higa Y., Tsuda Y., Suguyama A., Ishak H. Distribution and seasonal prevalence of the malaria vector mosquito, Anopheles minimus in Ishigaki Is., Ryukyu Archipelago, Japan, 1990-1994, Medical Entomology and Zo- ology, 1996; 47 63-72.

4 . Toma T., Miyagi I., Malenganisho W. L. M., Murakami H., Nerome H., Yonamine M. Distribution and seasonal occurrence of Anopheles minimus in Ishigaki Island, Ryukyu Archipelago, Japan, 1998-1999, Medical Entomology and Zoology, 2002; 53: Suppl.2, 29-42.

5 . Dietz K., Molineaux L., Thomas A. A malaria model tested in the African savannah, Bulletin of the World Health Organization, 1974; 50: 347-357.

6 . Garrett-Jones C. The human blood index of malaria vectors in relation to epidemiological assessment, Bulletin of the World Health Organization, 1964; 30: 241-261.

7 . Collett D., Lye M.S. Modeling the effect of intervention on the transmission of malaria in East Malaysia, Statistics in Medicine, 1987; 6: 853-861.

8 . Ishikawa H., Kaneko A., Ishii A. Computer simulation of a malaria control travel in Vanuatu using a mathematical model with variable vectorial capacity, Japanese Journal of Tropical Medicine and Hygiene, 1996; 24: 11-19.

9 . Nakazawa M., Ohmae H., Ishii A., Leafasia J. Malaria infection and human behavioral factors: A stochastic model analysis for direct observation data in the Solomon Islands, American Journal of Human Biology, 1998; 10: 781-788.

10 . Gu W., Killeen G. F., Mbogo C. M., Regens J. M., Githure J. I., Beier J. C. An individual-based model of Plasmodium falciparum malaria transmission on the coast of Kenya, Transactions of the Royal of Tropical Medicine and Hygiene, 2003; 97: 43-50.

11. Japan Meteorological Agency. Yearly meteorological data in Ishigaki Island (in Japanese). Available from http://www. data.jma.go.jp/obd/stats/etrn/view/annually_s.

12. Tsuda Y., Iizuka S., Haseyama M., Tajima S., Niizuma J., Yamauchi T. An occurrence of malaria vectors in Ishigaki Island. In: The report of the studies on monitoring an invasion of infectious diseases in Asian countries to our country, 2006 (Project 3), National Institute of Infectious Diseases, Tokyo, 2006; 347-353 (in Japanese).

13. Geographical Survey Institute, Government of Japan. Areas of islands in Okinawa, 2008 (in Japanese). Available from http://www.gsi.go.jp/KOKUJYOHO/MENCHO/200804/ shima/shima-oki.htm

14. Yaeyama Branch of the Prefectural Government of Okinawa. Yaeyama directory, 2008; 1-195 (in Japanese). Available from http://www3.pref.okinawa.jp/site/contents/attach/ 17935/H19-Y.youran-all.pdf

15. Department of Tourism, The Municipal Government of Ishigaki. Survey about the number of tourists at Ishigaki Airport and Ishigaki Port, 2006 (in Japanese). Available from http://www.city.ishigaki.okinawa.jp/110000/tourism/ kankouka/images/200812.pdf

16. Japan Travel Bureau Foundation Co. The study on a ripple effect of resorts in Yaeyama Archipelago, 2001, private communication (in Japanese).

17. Tourism Planning Division, The Prefectural Government of Okinawa. The survey on tourism statistics and satisfaction in tourists. In: The report on tourism statistics 2008 (in Japanese). Available from http://www3.pref.okinawa.jp/site 
/view/contview.jsp

18. Ueki M., Nakagawa Y., Fueda K., Ishikawa H. Statistical modeling for analysis of malaria epidemic behavior at Ishigaki Island, Journal of the Faculty of Environmental Science and Technology Okayama University, 2008; 13: 7-15 (in Japanese with English abstract).

19. Yaeyama Regional Public Health and Welfare Division. The history and result of malaria eradication program in Yaeyama Archipelago. In: The history and result of malaria eradication program in Yaeyama Archipelago, Yaeyama Regional Public Health and Welfare Division, 1965. In: The history in Ishigaki City, Ishigaki City Government, Ishigaki City, 1989: 753-791 (in Japanese).

20. Murakami H. The transition on malaria epidemic in Yaeyama Archipelago of Okinawa. In: The transition on malaria epidemic in Yaeyama Archipelago of Okinawa, Yaeyama Regional Public Health and Welfare Division, Okinawa prefecture, 1998; 1-6 (in Japanese).

21. Statistics Bureau, The Ministry of Internal Affairs and Communications, Japan. Basic Resident Register, 2007 (in Japanese). Available from http://www.city.ishigaki.okinawa. jp/100000/100300/toukei2007/toukei-pdf/2007\%2002.pdf

22 . World Health Organization, Report of the WHO valuation team on malaria eradication in the Ryukyu Islands. WPR/ 419/65. WHO, Geneva, 1966, pp.1-53

23. Eshita Y. The historical consideration of Malaria epidemic and its control project in Ryukyu Island. In: Environment and Sanitation (Kankyou-eisei), 1982; 29: In: The history in Ishigaki City, Ishigaki City Government, Ishigaki City, 1989: 846-856 (in Japanese).

24 . Warrell D. A., Clinical features of malaria, In: Essential malariology $4^{\text {th }}$ edition, Warrell D. A. and Gilles H.M. (Eds), Arnold, London, 2002, pp191-206

25 . Epstein J. E., Rao S., Williams F., Freilich D., Luke T., Sedegah M., de la Vega P., Sacci J., Richie J. T., Hoffman S. L. Safety and clinical outcome of experimental challenge of human volunteers with Plasmodium falciparum infected mosquitoes: An update, The Journal of Infectious Diseases, 2007; 196: 145-154.

26 . Smalley M. E., Sinden R. E. Plasmodium falciparum gametocytes: their longevity and infectivity, Parasitology, 1977; 74: 1-8.

27 . Morishita K. Epidemiology and prevention of malaria, Seison publishing company, Tokyo, 1976 (in Japanese).

28 . Earle W.C., Perez M., Del Rio J., Arzol C. Observations on the course of naturally acquired malaria in Puerto Rico, Puerto Rico Journal of Public Health and Tropical Medicine, 1939; 14: 391-406.

29 . van den Broek I., Kitz C., Attas S. A., Libama F., Balasegaram M., Guthmann J. P. Efficacy of three artemisinin combination therapies for the treatment uncomplicated Plasmodium falciparum malaria in the Republic of Congo, Malaria Journal, 2006; 5: 1-9.

30 . Bousema J. T., Schneider P., Gouagna L. C., Drakeley C. J., Tostmann A., Houben R., Githure J. I., Ord R., Sutherland C. J., Omar S. A., Sauerwein R. W. Moderate effect of Artemisinin-based combination therapy on transmission of
Plasmodium falciparum, The Journal of Infectious Diseases, 2006; 193: 1151-1159.

31 . Bousema J. T., Gouaga L. C., Drakeley C. J., Meutstege A. M., Okech B. A., Akim I. N., Beier J. C., Githure J. I., Sauerwein R. W. Plasmodium falciparum gametocyte carriage in asymptomatic children in western Kenya, Malaria Journal, 2004; 3: 1-6.

32 . Drakely C., Sutherland C., Bousema J. T., Sauerwein R. W., Taegett G. A. The epidemiology of Plasmodium falciparum gametocyte: weapons of mass dispersion, Trends in Parasitology, 2006; 22: 424-430.

33 . Christen D., Steffen R., Schlagenhauf P. Deaths caused by malaria in Switzerland 1988-2002, The American Society of Tropical Medicine and Hygiene, 2006; 75: 1188-1194.

34 . Yoshino K., Kuroshima T. Malaria epidemic in Yaeyama Archipelago at the war in 1945. In: The achievements in Public Health Department, Yaeyama Civil Government, Yaeyama Civil Government, 1947; In: The history in Ishigaki City, Ishigaki City Government, Ishigaki City, 1989: 694-735 (in Japanese).

35. Mckenzie F. E., Bossert W. H. The dynamics of Plasmodium falciparum blood-stage infection, Journal of Theoretical Biology, 1997; 188: 127-140.

36 . Bhargava A., Chatterjee B. Chikungunya fever, falciparum malaria, dengue fever, Japanese encephalitis . Are we listening to the warming signs for public health in India?, Indian Journal of Medical Ethics, 2007; 4: 18-23.

37 . Chalumeau M., Holvoet L., Cheron G., Minodier P., FoixL' Helias L., Ovetchkine P., Moulin F., Nouyrigat V., Breart G., Gendrel D. Delay in diagnosis of imported Plasmodium falciparum malaria in children, European Journal of Clinical Microbiology and Infectious Diseases, 2006; 25: 186189.

38. Centers for Disease Control, Taiwan, 2008 (in Chinese). Available from http://nidss.cdc.gov.tw/SingleDisease.aspx

39 . Infectious Disease Surveillance Center. Malaria in April, 1999-2005, Infectious Agents Surveillance Report, 2007; 28: 1-3 (in Japanese). Available from http://idsc.nih.go.jp/ iasr/28/323/tpc323-j.html 